Review Article

\title{
Venous Cerebral Infarction Caused by Intake of Hormonal Contraceptives in Adolescents with Congenital Thrombophilia
}

\author{
Putilina Marina $^{1}$, Teplova Nataliya ${ }^{1}$, Dvornikov Anton ${ }^{2}$ \\ ${ }^{1}$ Department of Clinical Pharmacology, Russian National Research Medical University Named After Pirogov, Moscow, Russian Federation \\ ${ }^{2}$ Department of Dermatology and Venereology, Russian National Research Medical University Named After Pirogov, Moscow, Russian \\ Federation
}

Email address:

profput@mail.ru (P. Marina), Teplova.nv@yandex.ru (T. Nataliya),dermatology@mail.ru (D. Anton)

\section{To cite this article:}

Putilina Marina, Teplova Nataliya, Dvornikov Anton. Venous Cerebral Infarction Caused by Intake of Hormonal Contraceptives in Adolescents with Congenital Thrombophilia. American Journal of Pediatrics. Vol. 6, No. 3, 2020, pp. 199-206.

doi: 10.11648/j.ajp.20200603.15

Received: April 18, 2020; Accepted: May 6, 2020; Published: May 15, 2020

\begin{abstract}
In recent years, an increase in dysmenorrhea among adolescents has been observed worldwide. Oral contraceptives are used in gynaecological practice to correct this condition. However, the prescription of drugs belonging to this group often misses the risk of developing vascular complications, such as cerebral venous thrombosis and cerebral venous infarction. Due to the growing percentage of patients with congenital genetic mutations, it is relevant and reasonable to consider the risk factors and clinical signs of venous infarction in adolescents. First, it is necessary to exclude congenital thrombophilia, factor V Leiden and prothrombin G20210A gene mutations, and deficiency of proteins C, S and antithrombin. Of particular notice are the young patients and adolescents with complaints of a first-time, intense, poorly treatable headache (or headache in anamnesis), changes in the eye fundus (edematous, hyperemic optic disks; dilated, plethoric veins in the fundus of the eye). In such cases, a thorough medical history is extremely important, including, in particular, detection of an infectious process, traumatic brain injury, venous thrombosis of any localization; the use of drugs (especially hormonal ones) that can provoke the development of a hypercoagulable state; and investigation of the family history. These measures will help to prevent development of a cerebral venous circulation disorder (venous infarction).
\end{abstract}

Keywords: Venous Infarction, Thrombophilia, Oral Contraceptives

\section{Introduction}

In recent years, an increase in dysmenorrhea among adolescents has been observed worldwide [1-2]. Oral contraceptives are used in gynaecological practice to treat this condition. However, prescription of drugs belonging to this group often fails to consider the risk of developing vascular complications, such as cerebral venous thrombosis and cerebral venous infarction (VI).

Venous infarction is considered to be a "relatively rare cerebrovascular disease", occurring in $0.5 \%$ to $5 \%$ of all cases of stroke [3]. Formerly, infectious and inflammatory processes of paranasal sinuses, otitis, mastoiditis, meningitis, septic conditions, and infection of the soft tissue of the face were considered to be the main etiological factors of cerebral venous infarction with thrombosis. However in recent years other conditions have come to the fore, specifically those that are in one way or another related to blood coagulation disorders (genetically determined and acquired coagulopathies) and to changes in the vascular wall, especially in female patients who use oral contraceptives (containing desogestrel or gestodene) [4]. According to the AHA / ASA data from 2011, hereditary thrombophilia, primary antiphospholipid syndrome, hyperhomocysteinemia, and the use of oral contraceptives were among the prothrombotic factors of cerebral venous sinus thrombosis (CVST) [3]. The difficulty of diagnosing VI in case of congenital pathologies 
follows primarily from the fact that the initial patient complaints are about headaches of various kind without pronounced focal neurological symptoms; as a consequence, there is a lack of clinical alertness for further diagnostic search [5]. Due to the growing percentage of patients with congenital genetic mutations, it is relevant and reasonable to consider the risk factors and clinical signs of the venous infarction in adolescents [6]. First, it is necessary to exclude congenital thrombophilia, factor V Leiden and prothrombin G20210A gene mutations, and deficiency of proteins $\mathrm{C}, \mathrm{S}$ and antithrombin.

\section{Risk Factors for Venous Infarction in Adolescents}

\subsection{Congenital Thrombophilia}

Thrombophilia is not a disease, but should be considered as a pathological condition (a propensity for thrombosis), caused by a combination of thrombogenic risk factors, and resulting in the development of thrombosis. The presence of thrombophilia is associated with an increased risk of pregnancy complications (habitual miscarriage, placental insufficiency, fetal growth restriction, late-stage toxicosis (gestosis)). The most pronounced activation of the thrombocytic hemostasis is observed in case of congenital hematogenous thrombophilia caused by the disruptions in the system of physiological anticoagulants. An increase in platelet aggregation function is observed in patients with thrombophilia resulting from hyperhomocysteinemia.

In 2013, J. Heit, in "Consultative Hemostasis and Thrombosis", provided the following classification of thrombophilia (or signs associated thereof), including both certain disorders in the hemostatic system, and a large number of pathological conditions, as well as the consequences of drug treatment (Table 1) [7].

Table 1. Classification of thrombophilia [7].

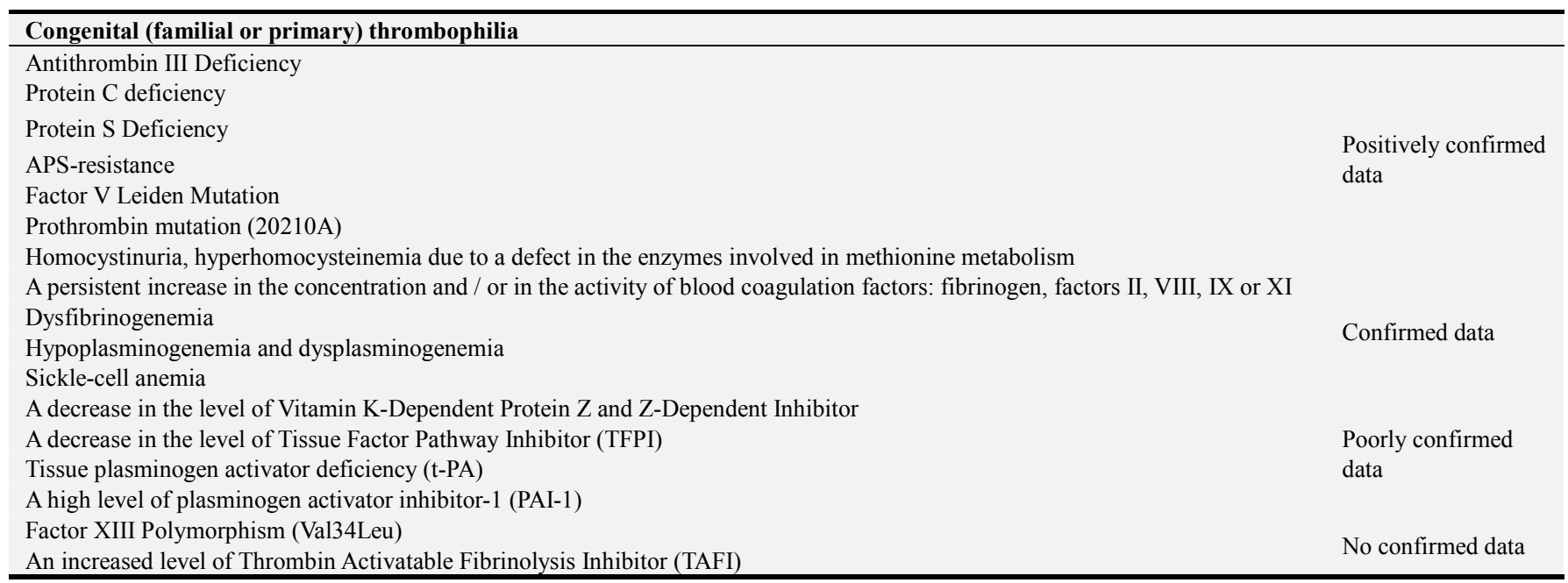

Genetic or acquired thrombophilia is the most common cause of cerebral venous thromboses and infarctions [8]. It should be suspected in adolescents with complaints of frequent "migraine-like" headaches, with dysmenorrhea, recurrent cerebral and extracerebral venous thromboses, provided there is an aggravated family history and no obvious intravital causes. The discovery of the following genetic mutations is crucial for the diagnosis of thrombophilia: Leiden and F2-prothrombin G20210A, MTHFR, PAI-I, factor VII, GP IIIa. [9-12].

\subsubsection{Factor V Leiden Gene Mutations}

This mutation occurs in $5 \%$ of people of the Caucasian race [9]. Patients who are both carriers of prothrombin F2 gene polymorphism and F5 polymorphism (Leiden mutation) are most at risk of developing thromboses and infarctions.

\subsubsection{Prothrombin G20210A Gene Mutation (Coagulation Factor II)}

F2 encodes a prothrombin protein, which is one of the main components of blood coagulation system. Under the influence of activated prothrombinase (FXa) in complex with activated factor $5(\mathrm{FVa})$ and calcium ions, prothrombin is converted into thrombin, which in return stimulates conversion of fibrinogen into fibrin, followed by formation of a blood clot. Polymorphism is passed onto the offspring with a probability of $50 \%$ [12]. Therefore, when a patient has this mutation, it is necessary to examine his immediate family to clarify their genetic profile and apply timely preventive measures against thrombosis. If this mutation is detected, substitution of oral contraceptives with other methods of contraception is recommended. If there is a history of previous thromboses, a lifelong treatment with anticoagulants is indicated. Anticoagulant treatment is also indicated during management of a complicated pregnancy. Individuals exhibiting this polymorphism have an increased risk of postoperative death due to thromboembolism. Genetic predisposition to thromboembolism must be taken into account when prescribing drugs to cancer patients.

Typical manifestations of prothrombin F2 gene polymorphism include:

1. Early venous and arterial thromboses, 
2. A positive family history of venous thrombosis,

3. Myocardial infarction, strokes,

4. Thromboembolic complications during pregnancy,

5. Pregnancy pathologies (infertility, gestosis, preeclampsia, premature detachment of a normally located placenta, habitual miscarriage, feto-placental insufficiency, intrauterine fetal death, fetal growth restriction).

\subsubsection{Mutation of Methylenetetrahydrofolate Reductase (M677T)}

The occurrence of M677T mutation ranges from 10 to about $40 \%$ [11]. Most frequently, the gene is found in Europeans, and least frequently in black Africans, and the natives of Australia and Sri Lanka. In Europe, the frequency of the 677T allele is the lowest in Scandinavians, and the highest in Southerners (residents of the Mediterranean). Regardless of the region, the presence of the $677 \mathrm{~T}$ allele is associated with an increase in plasma homocysteine; in homozygotes this increase is much more pronounced than in heterozygotes. The noted $677 \mathrm{~T}$ mutation predisposes to the development of moderate hyperhomocysteinemia, especially with an underlying decrease in folate status. Adolescents with 677TT genotype are prone to developing folic acid deficiency. In non-pregnant women, homozygous for this allele, folate deficiency can only be detected in red blood cells, while plasma folate level may stay unimpaired. However, during pregnancy, homozygous women experience a decrease in the concentration of folates not only inside red blood cells, but also in blood plasma.

\subsection{Antiphospholipid Syndrome (APS) As an Independent Risk Factor for $V I$}

APS is an acquired autoimmune process, which is based on the formation in the body (in a high titer) of biomodal autoantibodies that interact with negatively charged membrane phospholipids and glycoproteins which are linked to those. APS is a kind of vasculopathy caused by non-inflammatory and / or thrombotic lesions of the vessels and ending with their occlusion. In recent years, APS has been considered as a hypocoagulation form of the autoimmune hematogenous thrombophilia with hyperactivation of the immune system, of a possibly viral etiology, and with the circulation of IgG or IgM class antibodies in the blood [13]. It develops in young patients and middle-aged persons, while $85 \%$ of patients are in the age range from 15 to 50 years [14]. The ratio between men and women can vary depending on the disease form (1: 3.5 for primary APS and 1: 7 for secondary, associated with SLE). An increase in the incidence of antiphospholipid antibodies has been observed in some inflammatory, autoimmune and infectious diseases, malignant neoplasms, against the background of medication treatment (oral contraceptives, psychotropic drugs, etc.). There is evidence of an immunogenetic predisposition to the increased synthesis of antiphospholipid antibodies and its more frequent detection in relatives of patients with APS.

The development of antiphospholipid syndrome is associated with the damage to the central nervous system, cardiovascular system, impaired kidney and liver function, malfunctioning of endocrine organs and gastrointestinal tract. The damage to the nervous system may include transient ischemic attacks (TIA), ischemic stroke, ischemic encephalopathy, episyndrome, migraine, chorea, transverse myelitis, sensorineural hearing loss.

According to the modern classification, the following forms of APS are distinguished [15]:

Primary APS. Approximately one half of patients with APS suffer from the primary disease. However, the nosological independence of the primary APS is not fully understood. There is evidence that the primary APS can sometimes be a variation of the onset of SLE. Conversely, in some patients with early-stage classic SLE, signs of APS may manifest in the future. It is characterized by the post-thrombotic syndrome, habitual miscarriage or infertility, allergy to drugs (quinidine, hydralazine, phenothiazine);

Secondary APS in patients with a definite diagnosis of SLE. It is often found in autoimmune diseases (systemic lupus erythematosus (SLE), rheumatoid arthritis), malignant neoplasms, infectious and infectious-immune diseases (Lyme disease). With this type, the course, severity and prevalence of thrombotic complications are unpredictable and in most cases do not correlate with changes in antiphospholipid antibody titers and SLE activity;

\section{APS in patients with lupus-like manifestations;}

Catastrophic APS (acute disseminated coagulopathy / vasculopathy) with acute multi-organ thrombosis;

Other microangiopathic syndromes (thrombotic thrombocytopenic purpura / hemolytic-uremic syndrome); HELLP syndrome (hemolysis, increased activity of hepatic enzymes, decreased platelet count, pregnancy); DIC; hypoprothrombinemia syndrome;

Seronegative APS.

Certain manifestations, such as thrombocytopenia, livedo reticularis, valvular defect, nephropathy against the background of serological markers of APS, may signal the debut of the disease. At the moment, the presence of any of these symptoms with elevated levels of antiphospholipid antibodies in the absence of other evident causes is verified as "preAPS" or probable APS.

Clinical picture of APS

Headaches are traditionally regarded as some of the most common clinical manifestations of APS. The nature of headaches varies from a classic intermittent migraine to persistent, intolerable pain. Patients often have vein-occlusive eye diseases. Transient vision loss (amaurosis fugax) is sometimes found in this pathology. Another manifestation is the optic neuropathy, which is one of the most common causes of blindness in APS. Thrombocytopenia is typical. Usually, the platelet count ranges from 70 to $100 \times 109 / \mathrm{L}$ and does not require special treatment. The development of hemorrhagic complications is rare and is usually associated with a concomitant defect of specific coagulation factors, kidney pathology, or an overdose of anticoagulants. Coombs-positive hemolytic anemia (10\%) is often observed, Evans syndrome 
(a combination of thrombocytopenia and hemolytic anemia) is less common. Skin lesions in AFS are characterized by a variety of clinical manifestations, such as livedo reticularis, skin ulcer, pseudovasculitis and vasculitis lesions, Raynaud's syndrome and subungual infarctions ("splinter syndrome"). An increase in the level of antiphospholipid antibodies has been described in patients with Degos disease - a very rare systemic vasculopathy, manifested by common thrombosis of the skin, central nervous system and gastrointestinal tract.

\section{Venous Infarction in Patients with Thrombophilia}

Generally, venous infarction (VI) is associated with cerebral venous thrombosis. However, in adolescents and young patients under 45 , mainly women, it may develop without manifestations of the concomitant sinus thrombosis [16]. It is likely that endocrine factors play an important role in the pathogenesis of VI in women. Long-term use of oral contraceptives is a trigger for the development of venous pathology in patients with thrombophilia $[13,14]$. Symptoms of the disease, such as an increasing frequency and intensity of headaches may manifest in adolescents even without arterial hypertension and diabetes mellitus [16]. Headaches can be constant - 46.8\%, periodic - 51\%; diffuse - 51.8\%; local (mainly in the temporal regions, akin to hemicrania) $-46 \%$, intensifying after a long stay in a horizontal position, with a bowed head, when coughing, sneezing, straining. Patients may experience heaviness in the head, a sensation of pressure on the eyes from the inside, certain constrictions and pain when moving the eyeballs, "fullness" in the head, "machine" noise in the head (33.2\%), and irregular dizziness (32.4\%), syncopal episodes, decreased visual acuity, a sensation of "veil", "flies" before the eyes (19.1\%), sleep disturbance (50.1\%), feeling of a "tight collar" (38.2\%), hallucinations, nightmares, drowsiness, fatigue, autonomic disorders: "blood rushing to the head", palpitations, fear of death, shortness of breath, sweating, chills or hot flushes. During examination, swelling of the face has been detected (especially after sleep), less often the expansion of saphenous veins on the face and neck, edema of the conjunctiva and eyelids, scleral vascular injection; nosebleeds are possible with an intensifying headache. A decrease in headaches is typical after several days of heparin treatment [17]. In most cases of VI, on the 4th-5th day after the onset temperature increases to subfebrile digits. In almost one fifth of the cases, the development of convulsive seizures has been noted. A tendency towards rapid regression of cerebral and focal symptoms has been detected. Usually, focal migratory symptoms are observed in cases of cerebral involvement, i.e. a partial brain edema is followed by extensive cortical venous infarction [18].

\subsection{Verification of the Diagnosis of Venous Infarction}

Head MRI with MR venography is the method of choice for diagnosis verification; however these methods are not always available, especially in the acute phase. Generally, the first study in the case of a suspected stroke is computed tomography without contrast enhancement (CT scan - axial sections) [18, 19]. Unfortunately, CT often fails to detect thrombosis (in $25-40 \%$ of cases pathological changes in the brain tissue are not detected), especially if the study is carried out without contrast medium injection [15]. But it is possible to suspect the venous nature of an initially non-hemorrhagic stroke on the basis of its most pronounced symptoms: symptom of a hyperdense vessel on a routine CT scan; typical localization of the hypodense region in the parieto-occipital region and in the cerebellum with thrombosis of the upper sagittal, transverse and sigmoid sinuses, and also in the corpus callosum and in the thalamus with thrombosis of the lower sagittal and direct sinuses, of the internal veins of the brain outside any arterial pool; an irregular shape of the focus. A distinctive feature of a venous stroke consists in the signs of vasogenic edema (according to MRI) on the first day of the disease. The shape of cerebral infarction foci during the venous stroke is irregular, the contours are fuzzy and uneven, the MR signal in most cases is heterogeneous. Patients often show signs of cerebral venous dyscirculation and intracranial venous stasis [16]. As described above, the distinctive feature of VI is vasogenic edema on the first day of the disease. It is possible to accurately differentiate vasogenic edema from cytotoxic edema using such MRI modes as DWI (diffusion-weighted image) and ADC-mapping. Vasogenic edema is characterized by hypo- or iso-intensive foci on DWI, and an increase in signal intensity during ADC mapping. In case of an acutely developing intracranial hypertension syndrome, this symptom can be considered as a consequence of thrombosis of cerebral venous sinuses, provided that an increase in signal intensity in the projection of sinuses on T1-WI, T2-WI and FLAIR, is detected on MRI in the basic mode in the subacute period (from 5-7 to 30-35 days); sometimes the process may be accompanied by the development of venous infarction or edema in the brain substance located in the area of the thrombosed sinus.

To clarify the presence of hemorrhagic impregnation in the infarction zone, the inclusion of T2*-WI-based sequences in the study protocol (gradient echo mode: SWI, SWAN) is recommended. This allows determining the presence of hemorrhagic impregnation in the infarction zone in the early stages, as well as clarifying its presence in the focus of a previous cerebral infarction. The T2-WI sequence in conventional MRI can be used to calculate the area of brain damage in stroke, since it strongly correlates with the DWI and CBV map (quantitative analysis and color map of perfusion in cerebral blood volume mode), which are the markers of the final infarct size. Thus, the algorithm for neuroimaging diagnostics in patients with suspected VI should include: CT - in the acute period - in the first 5 days, basic MRI from the 5th-7th day - in the subacute period, and if necessary, MR angiography, MR venography or CT -angiography with a bolus administration of a contrast medium for the study of both arterial and venous phases of the cerebral blood flow. A laboratory study in cases of suspected VI should include determination of traditional biochemical 
parameters, homocysteine, genetic markers, coagulogram, a screening for APS [20]. According to the MEGA (Multiple Environmental and Genetic Assessment of risk factors for venous thrombosis) study, a screening for hereditary thrombophilia should take place if patients with a headache have an aggravated family history, helping to identify asymptomatic carriers of mutations, which will reduce or avoid (as is the case with oral contraceptives) additional risk factors for VI.

\subsection{Laboratory Screening for Suspected Thrombophilia}

Indications for screening include: suspected antiphospholipid syndrome; detection of thrombosis at a young age (up to 40 years); intake of oral contraceptives; pregnancy planning if there are immediate relatives who have had VTE; habitual miscarriage [21].

Following an algorithm for diagnosing hemostasis and monitoring antithrombotic therapy, laboratory screening should include:

1. In case of congenital thrombophilia associated with physiological anticoagulants, the determination of:

a) Antithrombin III;

b) Cofactor heparin II;

c) Protein C;

d) Protein $\mathrm{S}$.

2. In case of thrombophilia of autoimmune and infectious-immune genesis (under APS; immune thrombovasculitis; systemic immune diseases (Behcet's disease); hypertrophic cardiomyopathy; protracted immune endocarditis), screening for the underlying diseases.

3. In case of thrombophilia associated with changes in coagulation factors, screening for:

a) Factor $\mathrm{V}$ anomaly (Leiden mutation);

b) Mutations of prothrombin G202110A;

c) Deficiency of factor XII (prekallikrein);

d) Dysfibrinogenemia.

4. In case of thrombophilia associated with an increase or activation of plasma coagulation factors, the determination of coagulation factors VIII, VII, XI, IX, XIII.

It is important to bear in mind that during treatment of patients with protein $\mathrm{C}$ deficiency with indirect anticoagulants, they may experience skin necrosis as a result of rebound thrombosis.

To confirm the diagnosis of APS, the following laboratory criteria are proposed [22]:

a) Presence of lupus anticoagulant (LA);

b) Presence of antibodies to cardiolipin (aCL) of the IgG or IgM class in medium or high titer (more than 40 phospholipid units (PU) or in a titer exceeding 99 percentile) when using enzyme-linked immunosorbent assay (ELISA); $1 \mathrm{PU}$ is equal to $1 \mu \mathrm{g}$ of antibody;

c) Presence of antibodies to beta-2-glycoprotein of the $\mathrm{IgG}$ and / or IgM classes in a titer exceeding 99 percentiles, when using the ELISA method.

In recent years, numerous cases have been observed in clinical practice, which while clinically reminiscent of APS were negative according to the "standard" laboratory criteria (seronegative APS). Diagnosing APS in these patients is particularly difficult. It should be noted that the current criteria of the APS are mainly based on expert opinions rather than evidence-based research, so these should be treated critically. If the patient has clinical manifestations of APS, it is necessary to order tests for additional antiphospholipid antibodies that are not included in the accepted criteria:

a) Antibodies to the phosphatidylserine-prothrombin complex;

b) APA (antiphospholipid antibodies) of the IgA class. Currently, only IgG and IgM autoantibodies are counted. IgA antibodies are not taken into account. On the other hand, it has been shown that IgA-class antioxidants also increase the risk of thrombosis;

c) Antibodies to annexin V.

\section{Treatment of Venous Cerebral Infarction, Caused by the Intake of Hormonal Contraceptives}

The management of patients with arterial and / or venous thrombosis and AFL who do not exhibit the reliable AFS (serological markers at low levels) does not differ from the management of APA negative patients with similar thrombotic outcomes (level of evidence 1C). Patients with venous thrombosis, even without laboratory-confirmed APS, need treatment with anticoagulants. Usually, the initially prescribed heparins are: unfractionated (normal), or low molecular weight, or pentasaccharides, followed by a transfer to vitamin $\mathrm{K}$ antagonists (AVK) (warfarin).

Despite conducting anticoagulant therapy with warfarin (maintaining a medium-intense level of hypocoagulation, (2.0-3.0) and even high levels (above 3.0)), a relapse of thrombosis has been reported in some patients. Recommendations formulated by experts of the 13th International Congress for APA, offer low-molecular-weight heparins as an alternative to the long-term use of warfarin for such patients. Patients, who have been receiving a similar therapy for a long time, did not have a relapse of thrombosis. At the same time, all anticoagulants that have been in use for many years have several side effects and are far from "ideal" anticoagulants. It is believed that new anticoagulants with a selective point of application for coagulation factors will be more effective and safer than the heparins and warfarin. These new drugs, currently approved or being tested for the treatment and the prevention of venous thromboembolism, have a direct effect on the active site of the thrombin (a direct thrombin inhibitor) - dabigatran and direct factor Xa inhibitors: rivaroxaban, apixaban, edoxaban and betrixaban. Experts consider it reasonable to conduct randomized controlled studies of the treatment of APS with these drugs. The inclusion of hydroxychloroquine and statins in the treatment of such patients with APS is considered justified.

Patients with VI and established hereditary thrombophilia should be examined for deep vein thrombosis, the presence of which is an indication for a short-term or long-term 
anticoagulant therapy, depending on clinical and hematological parameters (class I, level of evidence A).

In patients with a history of thrombotic complications, a long-term anticoagulant therapy may be considered (class IIb, level of evidence $\mathrm{C}$ ). In the absence of venous thrombosis, a prolonged use of anticoagulants or antiplatelet therapy (class IIa, level of evidence C) is advisable [23, 24].

Although heparin is the drug of first choice (its effectiveness has been proved in controlled studies) [21], low molecular weight heparins (LMWH) have been increasingly used in the recent years: dalteparin, nadroparin, enoxaparin. The advantages of LMWH, compared to UFH, are a lower frequency of hemorrhagic complications, an extremely rare development of thrombocytopenia, a longer duration, high bioavailability, and the absence of need for frequent laboratory monitoring [22]. The therapeutic dose of enoxaparin is $1 \mathrm{mg} /$ $\mathrm{kg}$ of patient body weight bidaily. Furthermore, the transition to oral anticoagulants (warfarin, dabigatran, apixaban) is recommended. To date, the optimal duration of the anticoagulant therapy remains a debatable issue. In most recommendations for congenital thrombophilia, the administration of anticoagulants should last at least 6-12 months. Anticoagulants are prescribed for longer periods to patients with two episodes of thrombosis or with one episode of cerebral venous thrombosis provided that they have hereditary thrombophilia.

\section{A Clinical Case}

To illustrate the foregoing, we present a clinical case of a 16-year-old girl patient, who developed a venous infarction (against the background of hormone therapy), accompanied by a gradual intensification of the intracranial hypertension syndrome, cerebral and focal neurological symptoms.

Patient M., aged 16, visited a dermatologist with complaints of itching in the forearm, a pulsating headache, and general weakness. She associated the complaints with taking of oral contraceptives (Duphaston), prescribed by a gynecologist.

Anamnesis morbi: The patient has taken Duphaston $10 \mathrm{mg}$ 2 times/ day from the 5th to the 25th day of the menstrual cycle for 3 months, about 1 week ago started experiencing the itching in the forearm and a severe headache. Partial convulsions in the left limbs and the left half of the face started acutely. The ambulance emergency team was called, upon the arrival of which the symptoms regressed. Painkillers and dermatologist consultation are recommended. By the time of visiting the clinic, the headache had become constant, diffuse, unaffected by analgesics.

Anamnesis vitae: In childhood the patient experienced migraine-like headaches, increased fatigue, spasm in the throat, and a periodic sensation of not getting enough air. During the last year, she was under the care of a gynecologist regarding the irregular menstruation (lack of menarche). Receives hormone therapy (Duphaston). Mother suffers from migraines. The younger brother suffered an ischemic stroke at the age of 3 years. The patient's grandmother, aged 60 , and her father, aged 51, died of pulmonary embolism.
Status Present: height $170 \mathrm{~cm}$, weight $51 \mathrm{~kg}$. The skin is pale, dry, with no visible rashes, isolated antecedent hemorrhages. Severe cyanosis of the fingertips. The nails are dense, with cross-striation, there are white spots of 1-2 mm. The face is puffy, dark circles under the eyes. Signs of connective tissue failure: hypermobility of the joints, hyperelastic skin, tendency to mild bruising, valgus deformity of the lower extremities.

Recommended examination: clinical blood count with platelet count, determination of blood biochemical parameters: liver function tests, blood glucose, insulin resistance, glycated hemoglobin, C-reactive protein, homocysteine, coagulogram. Screening for APS. Blood culture tests. Consultations of a neurologist, ophthalmologist and hematologist.

Results of the examination:

1. Complete blood count: No pathology.

2. Urinalysis: no pathology.

3. Blood culture tests: there is no growth of aerobic and facultative anaerobic flora.

4. Biochemical blood test: no pathology.

5. Antibodies to phospholipids: antibodies to cardiolipin IgG 2.30 IU / ml, antibodies to cardiolipin IgM-not detected, antibodies to b2 glycoprotein I IgG 2.5 units / $\mathrm{ml}$, antibodies to b2 glycoprotein I IgM -not detected.

6. Blood coagulogram: APTT $27.3 \mathrm{sec}$, PT $11.5 \mathrm{sec}$, INR 1.05, PTI (prothrombin index) $100.0 \%$.

7. Consultation of an ophthalmologist: Signs of papilledema

8. Hematology consultation: Congenital thrombophilia? Genetic testing for gene polymorphism is recommended.

9. Genetic test for thrombophilic mutations: gene mutations of prothrombin G20210A, MTHFR, PAI-I, factor VII, glycoprotein IIIa were established.

10. A follow-up hematology consultation: Thrombophilia. Heterozygous polymorphisms (mutations) of genes: Prothrombin G20210A, MTHFR, PAI-I, factor VII, glycoprotein IIIa. Neurology consultation recommended.

11.USDG of the lower extremities veins: Echo-signs of thrombosis of deep and superficial veins of the lower extremities have not been detected.

12. Neurology consultation: the patient experiences an intense headache of diffuse nature, a sensation of pressure on the eyes from the inside, certain constrictions and pain when moving the eyeballs, a sensation of "fullness" in the head, a sensation of "veil", "flies" before the eyes, sleep disturbance, a sensation of a "tight collar" (38.2\%), constant chills, nosebleeds are possible with increasing headache drowsiness, clumsiness of the left hand which appeared 7 days prior to the consultation. During the examination, swelling of the face is observed, as well as edema of the conjunctiva and eyelids, scleral vascular injection; a slight asymmetry of the face is noted due to the smoothness of the left nasolabial fold. There is no paresis or paralysis. Performs co-ordinating tests. Diagnosis: Migraine with 
aura. Head MRI recommended.

13. Head MRI: Presentation of ischemic stroke in the left temporal lobe of the brain with hemorrhagic impregnation (venous type). Signs of decreased blood flow along the left transverse sinus. (Figure 1).
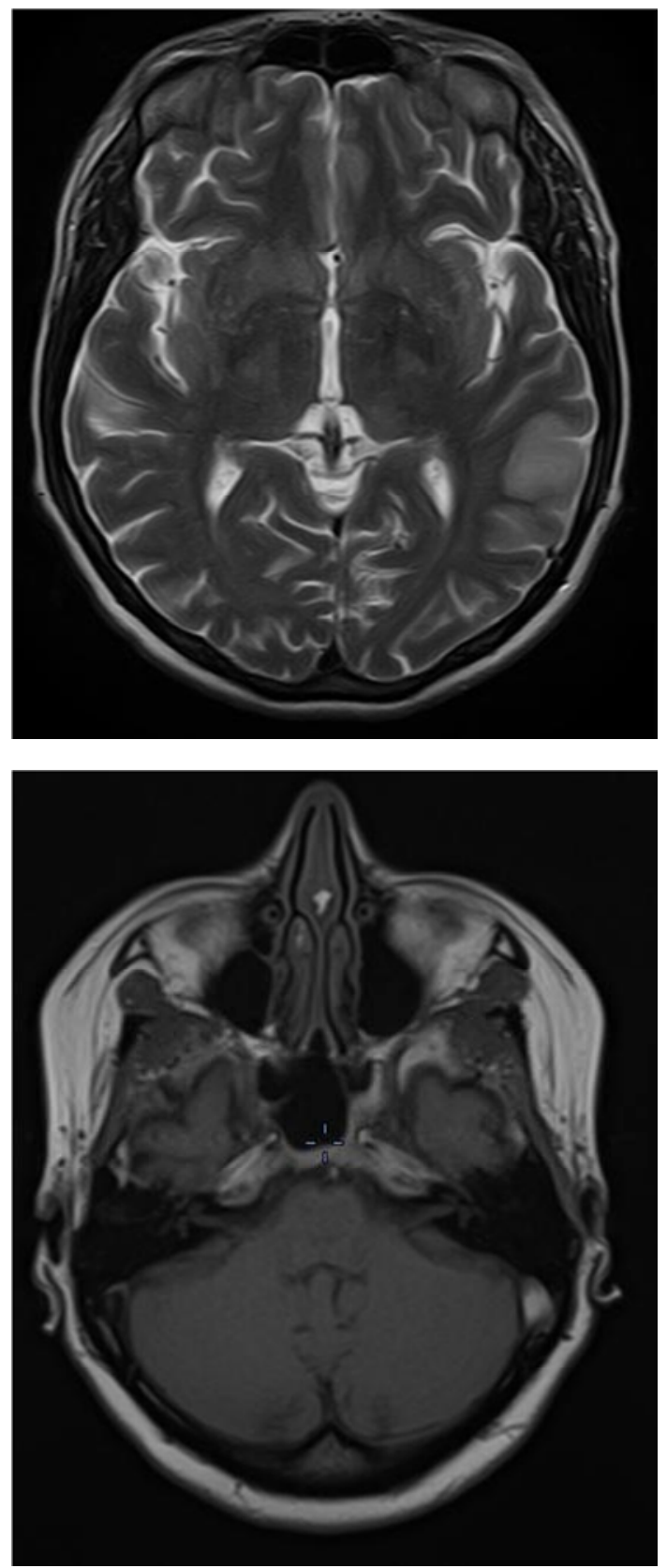

Figure 1. Head MRI of a patient M., 16 years old: Presentation of ischemic stroke in the left temporal lobe with hemorrhagic impregnation (venous type). Signs of decreased blood flow along the left transverse sinus.

14. A follow-up neurology consultation: the conclusive diagnosis (according to the neuroimaging data and taking into account the conclusion of the hematologist): Venous cerebral infarction in the left temporal region. Thrombophilia. Heterozygous polymorphisms (mutations) of genes: prothrombin G20210A, MTHFR, PAI-I, factor VII, glycoprotein IIIa.
The prescribed course of treatment:

1. Withdrawal of oral contraceptives.

2. Enoxaparin sodium $0.4 \mathrm{ml}$ x bidaily s / c - 2 courses ( 7 and 12 days); followed by switching to (apixaban) $5 \mathrm{mg}$ $\mathrm{x}$ bidaily for 6 months, then reducing the dose to $2.5 \mathrm{mg}$ bidaily; intake for a long period of time.

3. Detralex $500 \mathrm{mg} \times 2$ times a day. Prescribed for 6 months.

Re-examination by a neurologist after 6 months: no complaints. No abnormalities in the neurological status. It is recommended to discontinue anticoagulant therapy.

\section{Medical Conclusion}

Before prescribing medical hormonal correction to adolescents with menstrual irregularities, it is necessary to thoroughly screen for congenital changes in the body's coagulation system (thrombophilia, heterozygous polymorphisms of the genes: prothrombin G20210A, MTGFR, PAI-I, factor VII, glycoprotein IIIa, hyperhomocysteinemia, antiphospholipid syndrome). Of particular concern are the young patients and adolescents with complaints of a first-time, intense, poorly treatable headache (or headache in anamnesis), changes in the eye fundus (edematous, hyperemic optic disks; dilated, plethoric veins in the fundus of the eye). In such cases a thorough medical history is extremely important, and, in particular, the detection of an infectious process, traumatic brain injury, venous thrombosis of any localization; use of drugs (especially hormonal ones) that can provoke the development of a hypercoagulable state; and investigation of the family history. These measures should help to prevent development of a cerebral venous circulation disorder (venous infarction).

\section{References}

[1] Uvarova EV. Pathology of the reproductive system in childhood and adolescence. Gynecology. National leadership. A brief edition. Eds. Savelyeva GM, Sukhikh GT, Manukhina IB. M.: GEOTAR-Media. 2013; 186-193. (In Russ.).

[2] Bernardine H Stegeman, Marcos de Bastos, Frits R Rosendaal, A van Hylckama Vlieg, Frans M Helmerhorst, Theo Stijnen, Olaf M Dekkers. Different combined oral contraceptives and the risk of venous thrombosis: systematic review and network meta-analysis. BMJ 2013; 347 doi: http://dx.doi.org/10.1136/bmj.f5298 (Published 12 September 2013). Cite this as: BMJ 2013; 347: f5298.

[3] Saposnik G., Barinagarrementeria F., Brown R. D., Bushnell C. D., Cucchiara B., Cushman M., deVeber G., Ferro J. M., Tsai F. Y; American Heart Association Stroke Council and the Council on Epidemiology and Prevention. Diagnosis and management of cerebral venous thrombosis: A statement for healthcare professionals from the American Heart Association/ American Stroke Association. Stroke. 2011; 42: 1158-1192. doi: 10.1161/STR.0b013e31820a8364.

[4] Makarova T. A., Sotnikov A. Levin O. S. Venous thrombosis of the transverse sinus with hemorrhagic infarction and pure alexia is presented J. Nevrol. 2013; №4: 23: 29-33. (In Russ.)]. 
[5] Amoozegar F., Ronksley P. E., Sauve R., Menon BK. Hormonal contraceptives and cerebral venous thrombosis risk: a systematic review and meta-analysis. Front. Neurol. $2015 \mathrm{Feb}$ 2; 6: 7. (epub ahead of print). doi: 10.3389/fneur. 2015.00007.

[6] Fedin A., Ermoshkina N., Putilina, M., Vasilyev, Yu., Kozlov, M., Sidelnikova L., Snigireva T. Peculiarities of clinics and diagnosis of cerebral venous thrombosis // Clinical physiology of blood circulation, 2014: No. 1: 32-43. (In Russ.).

[7] Heit John A. Thrombophilia: Clinical and Laboratory Assessment and Management. / John A. Heit // Consultative Hemostasis and Thrombosis: Third Edition. Elsevier Inc. 2013. - p. 205-239.

[8] Garcia-Horton A, Kovacs MJ, Abdulrehman J, Taylor JE, Sharma S, Lazo-Langner A. Impact of thrombophilia screening on venous thromboembolism management practices. Thromb Res. 2017 Jan; 149: 76-80. doi: 10.1016/j.thromres.2016.11.023. Epub 2016 Dec 1.

[9] Dag Z. O. I., Işik Y. I., Simsek Y. I., Tulmac O. B. I., Demiray D. I. HELLP Syndrome and Cerebral Venous Sinus Thrombosis Associated with Factor V Leiden Mutation during Pregnancy//Case Rep Obstet Gynecol. 2014; 2014: 582-589. doi: $10.1155 / 2014 / 582890$.

[10] Liew SC, Gupta ED. Methylenetetrahydrofolate reductase (MTHFR) C677T polymorphism: epidemiology, metabolism and the associated diseases. Eur J Med Genet. 2015 Jan; 58 (1): 1-10. doi: 10.1016/j.ejmg.2014.10.004. Epub. 2014 Nov 4.

[11] Kang S, Zhao X, Liu L, Wu W, Zhang D. Association of the C677T polymorphism in the MTHFR gene with hemorrhagic stroke: a meta-analysis Genet Test Mol Biomarkers. 2013 May; 17 (5): 412-7. doi: 10.1089/gtmb.2012.0295. Epub 2013 Feb 21.

[12] Sodavarapu S., Ali SW., Goyal M. Cerebral Venous Sinus Thrombosis with Bilateral Abducens Palsy in a Patient with Heterozygous Prothrombin G20210A Mutation. Cureus. 2019 Nov 11; 11 (11): e6124. doi: 10.7759/cureus.6124.

[13] Putilina M. V. Endothelium as a target of new therapeutic strategies in vascular diseases of the brain. Journal of neurology and psychiatry. S. S. Korsakova, 2017; 117 (10): 122-130. (In Russ).

[14] Madison JA1, 2, 3, Duarte-García A4, 5, 3, Zuo Y1, Knight JS1. Treatment of thrombotic antiphospholipid syndrome in adults and children. Curr Opin Rheumatol. 2020 Mar 13. doi: 10.1097/BOR.0000000000000702. [Epub ahead of print].

[15] Chaturvedi S., McCrae K. R. Diagnosis and management of the antiphospholipid syndrome. Blood Rev. 2017 Nov; 31 (6): 406-417. doi: 10.1016/j.blre.2017.07.006. Epub 2017 Jul 30.
[16] Semenov S. E., Moldavskaia I. V., Yurkevich E. A., Shatokhina M. G., Semenov A. S. Diagnosis of venous ischemic stroke. Part I (Clinical polymorphism). A review article. Complex Issues of Cardiovascular Diseases. 2019; 8 (2): 125-134. DOI: 10.17802/2306-1278-2019-8-2-125-134.

[17] Ramsey R. R., Ryan J. L, Hershey A. D, Powers S. W, Aylward B. S, Hommel K. A. Treatment adherence in patients with headache: a systematic review. Headache. 2014 May; 54 (5): 795-816. doi: 10.1111/head.12353. Epub 2014 Apr 17.

[18] Ryskeldiyev N. A., Zhumadildina A. Zh., Teltayev D. K., Olenbai G. I., Mustafin Kh. A. Dural plasty in the posterior cranial fossa. Neyrokhirurgiya i nevrologiya Kazakhstana 2013; 4 (33): 18-22.

[19] Auezova R. Zh., Moldabekov A. Ye., Tleubergenov M. A., A. K., Doskaliyev A. Zh. Brain Venous infraction after convexital meningioma excision J. Neurosurgery and neurology of Kazakhstan 2014 №1 (34): 20-24.

[20] Mashin V. V., Belova L. A., Moiseev M. YU., Proshin A. N. A comparative clinical and neuroimaging study of venous and arterial strokes. Annals of Clinical and Experimental Neurology. 2015; 4: 24-31. (In Russian).

[21] Crous-Bou M., Harrington L. B., Kabrhel Ch. Environmental and genetic risk factors associated with venous thromboembolism Semin Thromb Hemost. 2016 Nov; 42 (8): 808-820. Published online 2016 Oct 20. doi: $10.1055 / \mathrm{s}-0036-1592333$.

[22] Tasdemir S, Erdem HB, Sahin I, Ozel L, Ozdemir G, Eroz R, Tatar A. Correlation with Platelet Parameters and Genetic Markers of Thrombophilia Panel (Factor II g. 20210G>A, Factor V Leiden, MTHFR (C677T, A1298C), PAI- 1, $\beta$-Fibrinogen, Factor XIIIA (V34L), Glycoprotein IIIa (L33P)) in Ischemic Strokes. Neuromolecular Med. 2016 Jun; 18 (2): 170-6. doi: 10.1007/s12017-016-8386-x. Epub 2016 Mar 7.

[23] Antiphospholipid Syndrome: Insights and Highlights from the 13th International Congress on Antiphospholipid Antibodies D. Erkan and S. S. Pierangeli, DOI 10.1007/978-1-4614-3194-7_17, Springer Science+Business Media New York 2012.

[24] Prevention of VTE in Nonorthopedic Surgical Patients Antithrombotic Therapy and Prevention of Thrombosis, 9th ed: American College of Chest Physicians Evidence-Based Clinical Practice Guidelines. CHEST 2012; 141 (2) (Suppl): e227S-e277S.

[25] VTE, Thrombophilia, Antithrombotic Therapy, and Pregnancy: Antithrombotic Therapy and Prevention of Thrombosis, 9th ed: American College of Chest Physicians Evidence-Based Clinical Practice Guidelines. Chest. 2012 Feb; 141 (2 Suppl): e691S-736S. 